\title{
A REGIONAL SERVER FOR MEDICAL INFORMATION
}

\author{
* Nathalie Bricon-Souf, Ph.D (1), David Delerue (1), Hassan Bezzazi (1), \\ Didier Donsez, Ph.D. (2), and Régis J. Beuscart, M.D., Ph.D.(1,3),
}

\author{
1. CERIM. Faculté de Médecine - 1 Place de Verdun - 59045 LILLE Cedex (France) \\ 2. LIMAV, Université de Valenciennes - Le Mont Houy, BP 311, 59304 VALENCIENNES Cedex \\ (France) \\ 3. C.H.R.U. de Lille - 2, Avenue Oscar Lambret - 59037 LILLE Cedex, (France) \\ *E-mail : souf@univ-lille2.fr
}

\begin{abstract}
This paper describes the design of a modular architecture for servers dedicated to share medical information between health care partners within the same regional area: hospitals, laboratories, general practitioners, etc... This architecture uses some results of the European project ISAR-Telematics [1]. It is used itself as a model to implement a first server for the North - Pas de Calais Region in France within the framework of the IRISI projects [2]. These servers allow Medical Information to be transmitted between different Medical Information Systems of various types (HIS, GPS' Software, Laboratories' Information Systems) which were not designed to satisfy inter-operability problems. This architecture is based on widely used technologies like $W W W$.
\end{abstract}

\section{Introduction}

Today, the evolution of Healthcare Systems is of interest to all countries in Europe. Currently, European countries are getting equipped with an infrastructure of Telecommunication that will allow the Telematics services unfolding on a large scale. Many of them are establishing specific networks to Healthcare. This will allow them to take into account the step towards the Information Society using appropriate answers to their healthcare system.

The ISAR-T project is essentially a Research Programme within the "AIM - HealthCare Telematics" Programme. It is part of the strategy of European hospitals located in 5 different countries : Courtrai, Tournai (Belgium), Turku (Finland), Lille (France), Nijmegen (the Netherlands) and Lisbon (Portugal).

The aims of ISAR-T were to show how on an European level, between countries with very different HealthCare Systems and also really different level of computerisation, it is possible to :

- define a set of information and communication services between hospitals,
- demonstrate the transferability of these services from one country to another or from a medical area to another one (inside the European Union),

- demonstrate that common methodologies dealing with the evaluation of ergonomic, technical and economic issues can be applied and validated in this context.

The Regional University Hospital of Lille, one of the biggest French Hospital, anticipated the way to ensure the continuity of cares within it's borders through a full Hospital Information System. This entity is a now a leader for several experiments of the distribution of Information phase the "Computer-Aided-Society » has entered in... These projects deal with :

- EDI between ( Hospital and Hospital ) or (Hospital and General Practitioners) or (Laboratories and Hospitals)

- remote access to HIS for specialists in AIDS home care,

- training or diagnoses using video-conferencing systems,

To reduce redundancy of resources, to enhance quality of common services, to reuse from one project with another, these experiments are connected to the same server considered as « Regional ». The effective size of the region concerned is not clearly defined yet but the genericity of the server designed will allow very different configurations.

\section{Goals}

The server will be tested in several places, the modification of the activities, the correctness of services delivered, the economical impact on its use will then be carefully evaluated and allow incremental improvements.

After a global evaluation of these results, this experiment will hopefully be distributed widely. Nevertheless, the first attempts show that it really takes a lot of time to organise these changes. Main issues that must be sorted are :

- infrastructure problems,

- users training,

- changes in the organisation,

- guidelines definition,

- the possibility to integrate new products, 
and of course technical choices.

\section{Methodologies}

The Users' Requirements Analyses were performed using DIGITAL methodologies : wall-charting and top-mapping. The results were formalised using an extension of the KAVAS methodology developed previously [3] in a ( Problem / Cause / Consequence / Expectation / Suggestion / Priority) form.

The results of these Users Needs Requirement [4] clearly show that whatever the systems are from a very global point of view, the main problems are due to a lack of Information exchange.

\section{Users Needs Requirements selected}

In the first stage of the project, we have selected the functionalities which have the top priority according to the GPs. These first functionalities to be implemented are :

- Admission, Discharge, Transfer events communication

- laboratory results transmission : the laboratory from the hospital and the private laboratories may transmit results easily in France because a standard is used for this (HPRIM),

- information access

- using services like :

- security management,

- directories,

- electronic mail,

- remote connection

\section{Services Identified}

Main services expected in a Regional Server for Medical Information are :

- infrastructure management in all aspects: Hospital LAN to LAN interconnection, access server, administration of the server,

- mail services: mail account attribution, gateways to specific mailing systems,

- directory management: to define, administrate, maintain directories for healthcare professionals is a key activity ; a failure in the directory use is definitely a reason of giving up such a regional server,

- security management in all aspects: confidentiality, certification, non-repudiation, data integrity,

- profiles configuration : to allow different notification, specify which kind of software is used, describe mailing system,

- information repository,

- conversion tools,

- distributed medical record database,

- co-operation medical protocols

\section{Constraints}

Legal issues which apply to data privacy as well as security mechanisms are a serious problem to this kind of project but these problems can be ignored in an experimentation where it is possible to use simulated data.

\section{Conclusion and Perpectives}

Some results of ISAR-Telematics prototype are taken into account to build a Regional Server for Medical Information in the Nord - Pas de Calais Region (France). Gathering results of other experiments from European partners Nijmegen (Holland), Tournai(Belgium) it will be possible to implement a kernel of generic services offering the basic services expected by Healthcare actors. Therefore, a global evaluation, a comparison with other convergent approaches should help the Industrialisation of these processes towards some kind of "Healthcare Society". These services will contribute to a better co-ordination of care by means of a better continuity of information.

\section{Acknowledgements}

This project is supported by DGXIII of European Union Health Telematics Programme (HC1027), by the European IRIS Initiative, and the Ganymede CPER of the Northern Region of France.

\section{References}

1. D. Donsez, G. Tiers, B. Modjeddi, R.J. Beuscart, "Improving the Continuity of Care: The ISAR Telematics European Project », in proceedings of 1996 AMIA Annual Fall Symposium, October 1996, Washington DC, p890.

2. N. Souf, G. Tiers, D. Donsez, R.J. Beuscart , «The Inter-Regional Information Society Initiative (IRIS): The Healthcare Working Group », in proceedings of 1996 AMIA Annual Fall Symposium, October 1996, Washington DC, p889.

3. Régis J. Beuscart, M.D., Ph.D., Sylvie Molenda, Nathalie Souf , Claude Foucher, M.D., MarieCatherine Beuscart-Zephir, "Assessment of a Cooperative Workstation », in proceedings of 1996 AMIA Annual Fall Symposium, October 1996, Washington DC, p742-746.

4. ISAR Telematics - HC 1027, Deliverable 2.1 : Common users' requirements methodology, September 96 